\title{
Interstate health-information exchange: understanding value propositions and challenges
}

This article was published in the following Dove Press journal:

Innovation and Entrepreneurship in Health

22 April 2016

Number of times this article has been viewed

Cynthia M LeRouge'

Joanna L Hirsch ${ }^{2}$

Sue S Feldman ${ }^{3}$

Angie L Bass ${ }^{4}$

'Department of Health Services, School of Public Health, University of Washington, Seattle, WA, ${ }^{2}$ State of Illinois Appellate Judicial System, Collinsville, IL, ${ }^{3}$ Department of Health Services and Policy Management, Arnold School of Public Health, University of South Carolina, Columbia, SC, ${ }^{4}$ Missouri Health Connection, Columbia, MO, USA
Correspondence: Cynthia M LeRouge Department of Health Services, School of Public Health, University of Washington, 1959 Northeast Pacific Street - Box 357660, Seattle, WA 98195-7660, USA $\mathrm{Tel}+\mathrm{I} 3147090624$

Fax +I 2065433964 Email lerouge@uw.edu

\begin{abstract}
Regional intrastate and statewide health-information exchanges help to connect patients' disparate intrastate electronic health records. Intrastate electronic health-information exchange is necessary, yet it does not sufficiently serve the needs of a mobile population, expanding health systems, or a concentration of specialized providers in urban centers serving geographically distant patients. The purpose of this multicase study, consisting of interview data collected from seven states and an intrastate health-information exchange literature review, is to explore the value propositions and challenges for connecting states through interstate exchange. Findings show that attention must be placed on expanding the interstate exchange of patient health data, as 1) many health systems cross state lines, 2) many providers serve patients in different states, and 3) patients are increasingly willing to travel long distances to receive specialized medical attention. An extended discussion contextualizes the findings in consideration of national health-information exchange.
\end{abstract}

Keywords: direct exchange, query-based exchange, electronic health record

\section{Introduction}

Accurate, available, and current data are "the lifeblood of health care improvement" and crucial in making health care more efficient, thorough, and affordable for people and communities. ${ }^{1}$ The electronic exchange of patient data gives health care providers access to the most up-to-date, aggregated data for making informed medical decisions. Currently, patient health data are not functioning efficiently for patients, as many health care professionals provide services without aggregated patient history. ${ }^{2}$ Modern health care is not a sole-source environment. Health care consumers have become mobile, increasing the need for quick access to reliable information in different locations. ${ }^{2}$ This can be a challenge, given the significant technical hurdles associated with patient-record identification, the exchange of data among differing electronic health record (EHR) systems across varying locations, and the dissimilation of useful information among providers. ${ }^{3}$

Intrastate (regional and statewide) health-information exchanges (HIEs) that achieve critical mass may help address the current fragmentation of patient health records. Expanding health systems and concentrations of specialized providers in urban centers geographically distant from the patients they serve extend the case for exchange to interstate exchange (ISE). ISE, the electronic exchange of patient data across two or more state borders, brings together many different stakeholders, including hospitals, state Medicaid programs, pharmacies, laboratories, imaging centers, nursing facilities, and other health care providers. It is expected that ISE will increase the coordination submit your manuscript

Dovepress f th in

http://dx.doi.org/| 0.2147/1/EH.S70055
Innovation and Entrepreneurship in Health 2016:3 69-77

(c) (i) (5) 2016 LeRouge et al. This work is published and licensed by Dove Medical Press Limited. The full terms of this license are available at https://www.dovepress.com/terms. (c) you hereby accept the Terms. Non-commercial uses of the work are permitted without any further permission from Dove Medical Press Limited, provided the work is properly attributed. For permission for commercial use of this work, please see paragraphs 4.2 and 5 of our Terms (https://www.dovepress.com/terms.php). 
of care across a large network of providers, decreasing costs, synthesizing fragmented care, and improving communication quality among health care professionals. ${ }^{4}$ In fact, the literature reveals that connectivity among providers on a large scale can facilitate the collection and timely dissemination of data in a confidential manner, decreasing the need for human interaction and thus improving response times in diagnosing and providing patient care. ${ }^{5}$ Given the increasing use of remote monitoring, the use of longitudinal records, especially with an ever-aging elderly population, strongly supports HIE. ${ }^{6}$

The value propositions of localized, state-level HIEs are currently being explored in practice $;^{7}$ however, to improve ongoing ISE examination specific to ISE design, implementation, utilization, and research outcomes are vital in understanding fully how the electronic exchange of patient data across state lines will improve health care quality in the modern, mobile, and expansive health care landscape. To contribute to ISE understanding, the purpose of this study is to explore the benefits, challenges, and potential strategies specific to ISE. Specifically, this study seeks to address the following research questions:

- What are the ISE value propositions and associated use cases?

- What are the ISE challenges and how can they be addressed?

The qualitative findings of this study can expand our understanding of the value propositions, challenges, and strategic solutions regarding the connection of states through ISE. Additionally, we discuss our findings as they relate to the efforts of national HIE. This study is contextualized to the USA. The complexities of the US health care system and nuances of state law make this a particularly interesting context of study.

\section{Materials and methods}

To gain a thorough understanding, a cross-state case study was deployed. This process consisted of key informant interviews, as well as an analysis of available popular press, gray, and academic literature to supplement these findings.

This evaluation was not subject to a consent or review process per the Institution Review Board officers. This was because the primary purpose of the evaluation was to fulfill the Missouri Health Connection's evaluation requirements, rather than a research focus.

We conducted semistructured interviews with nine key informants representing HIE networks from seven different states: Colorado, Iowa, Illinois, Kansas, Nebraska, Oklahoma, and Utah (representing 14\% of the USA). We chose to focus our data collection on states with multiple neighboring states to address the proposed research questions and for insight into ISE. Therefore, "multinexus" states were chosen to explore multiple exchange possibilities across state lines and to acknowledge multistate-exchange complexity. For example, Missouri has multiple nexuses with eight neighboring states, affording an opportunity to explore ISE from an octagonal perspective. We also interviewed key informants from two non-Midwestern states, as we were limited with the number of states to interview, to assist in easing potential geographical bias.

Nine 60-minute structured telephone interviews with audio recording were conducted with key informants to explore value propositions and obtain suggestions (beyond information publicly available) for meeting challenges. We selected interviewees based on their roles in leading respective state HIE initiatives. It is of note that when interviewees were asked if there was anyone in other states who could serve as a key informant for the process, references were made to one or more members of our interview panel.

Engaged interviewees were informed that the interview's purpose was to understand varying stakeholder factors and value propositions relative to HIE and ISE. The interview protocol was inspired by existing literature on HIE, as well as the program-information notice released by the Office of the National Coordinator in 2012. The following list comprises eight key interview questions used in this study:

- What benefits and barriers are associated with ISE?

- What criteria are most important in deciding with which states to connect?

- What are the value propositions and associated use cases?

- Does your state have a clearly identified utilizationpurpose policy?

- How will you determine to which state's privacy laws your state will adhere?

- Do you have concerns regarding security and privacy?

- What do you view as technical challenges?

- What do you believe are the most valuable strategies to facilitate implementation?

Data analysis involved two stages: coding of interview and supporting data, and a literature review of ISE to supplement understanding. We first coded the interview transcripts using Dedoose qualitative software to identify themes across respondents. In general, we used guiding principles from Lee and Baskerville ${ }^{8}$ to move data to description and thereby develop comprehensive understanding and insights. Two researchers independently open-coded the transcripts and any documents provided by interviewees to identify and 
define themes related to ISE value propositions, use cases, challenges, and strategies to address identified challenges (see Strauss and Corbin ${ }^{9}$ for detailed discussion of open-coding process). After independent open coding was complete, reliability and validity of the codes were established through a consensus-building process. Specifically, the research team (two coders and a third team member acting as an independent arbitrator) reviewed and discussed independent coding and supporting excerpts from the transcripts until a consensus on the themes was reached. When the themes became stable and the researchers reached consensus, the data analysis of the interviews and related documents was complete.

Following analysis of interview data and supporting documents, an analysis of available literature on ISE was conducted to expound on data obtained during the interview process and facilitate reliability and validity through data triangulation of multiple sources of information. Data sources included studies and reports concerning ISE, related state websites, and gray literature. The literature supplemented the interviews by providing further description, particularly providing an indication of the potential challenges ISE could face as connectivity crosses state lines among many different providers and provider organizations. We also looked for supporting evidence related to strategies proposed by interviewees. It is of note that this analysis produced a sparse listing of peer-reviewed literature principally focused on ISE (using search terms including ISE, cross-state exchange, and other synonyms with HIE-related terms), reinforcing the need for further ISE research in this area.

\section{Results}

\section{Value propositions and associated use cases}

Key informant interviews and available literature revealed common themes regarding the advantages of ISE. Collectively, these advantages provide benefits to participating providers and provider organizations.

\section{Increased sharing of best practices nationwide}

As represented among all interviewees, there is a hope that as more providers and health care systems become connected, ISE can facilitate the dissemination of best practices and known-use cases nationwide. In this regard, many interviews revealed that some leaders and providers have formed groups with other similarly situated states, which provide a public forum for involved HIEs to share operational best practices, as electronic health exchange further develops. One participant described the purpose of the Mid-States Consortium, a group with 23 HIE members from 17 Midwestern, South- western, and Western states, as "[a] group to set the standards for what is going to be acceptable for data sharing across the middle states of [the] country."

Additionally, the Strategic Health Information Exchange Collaborative, consisting of 34 HIEs representing 23 states, was recently formed. ${ }^{10}$ The purpose of the collaborative is to exchange business and strategic ideas among partners. ${ }^{10}$

\section{Utilization of referrals across state lines}

One interviewee provided a shared-use case that focused on how ISE is helping specialty and primary care providers enhance practice through increased referrals across state lines. Providers are using ISE connections to gain referrals and build practice volumes, thereby enhancing their competitive advantage. ISE offered providers the opportunity to refer their patients to a more comprehensive list of connected ISE health care professionals.

\section{Marketing tool to increase state HIE participation} Interviewees suggested that ISE would encourage nonparticipants to join local exchanges and benefit from increased access. Even though interviewees indicated that many of the larger hospitals were already participants within each respective HIE, a general sentiment among interviewees was that ISE agreements provided a good strategy to increase participation in the state HIE and were beneficial from a marketing standpoint.

\section{Expanded communication among ISE providers}

Interviewees indicated that ISE offered patients the ability to travel far from home with the confidence that providers could access their data and communicate as needed; therefore, ISE provided a platform for a patient's record to follow the patient, either through a provider's efforts to make a data request from a fellow provider ("push") or autonomously through the EHR system ("pull"). ${ }^{5}$ The general expectation among interviewees was that ISE would eliminate barriers to the provision of comprehensive care when medical emergencies occur far from home, or when patients travel to doctors across state lines. One interviewee articulated this hope as follows:

Patients can go back and forth between facilities and their providers $[\ldots]$ can look at that information and $[\ldots]$ already have a heads-up on that patient's health status before seeing the patient.

Informants commented that ISE offered providers a greater opportunity to collaborate with a patient's entire team of health care professionals - primary care and specialty 
care providers, caseworkers, psychiatrists, etc - who were working toward the same goal(s) for the patient. A common sentiment among interviewees was that the availability of extensive data through ISE allowed physicians at both ends to look up patient information electronically without having to rely solely on the patient's memory.

\section{Increased access to longitudinal care records}

Interviewees mentioned that the availability of records with a quick "pull" or "push" of the HIE network offered providers a more complete idea of a patient's overall health status. The idea is to assist in the transformation of health care delivery from treating only a specific symptom to treating the whole patient. One interviewee explained,

A provider may have an office in [state $\mathrm{X}$ ], but also have an office in [state Y]. It's important that [state $\mathrm{X}$ and state $\mathrm{Y}$ ] connect, so if a patient decides to go to a hospital in [state Y], the [complete] record is also available to the provider in [state $\mathrm{X}]$.

\section{Focus on public health reporting}

ISE implementation can facilitate the transmission of quality reporting to central registries (eg, certain cancers and birth defects). One interviewee noted that an ISE focus on public health allows

doctors to identify certain populations that might be more prone to certain conditions and then determine how to preemptively decrease or prevent these conditions, such as obesity and diabetes.

Furthermore, this respondent noted that

taking clinical information in the context of cultural and geographical circumstances is important, because it allows for data comparisons to determine the similarities and differences between states which will help to further population health-management efforts.

\section{Challenges and strategies}

Although many value propositions exist, key informant interviews and available literature revealed several barriers to overcome during the implementation and ongoing maintenance of ISE. We present these challenges later, as well as insight from our data sources regarding how these challenges can be addressed.

\section{Challenge I: Differing privacy laws}

The challenge of differing privacy laws was expressed throughout the interviews, supporting documents, and litera- ture. Interviewees indicated privacy of patient health data is a major legal issue with respect to ISE and HIE. The Health Insurance Portability and Accountability Act allows states the autonomy to determine the stringency of privacy with regard to certain health data, such as mental health, HIV testing, and substance abuse. ${ }^{11}$ As states have recently legislated laws concerning the use and disclosure of patient data, fragmentation among state laws has increased. ${ }^{12}$ Variations in state laws concerning terms, definitions, content, and organizational structure may be the most significant obstacles to implement and utilize ISE successfully. ${ }^{13}$

\section{Potential strategies to overcome challenge I:}

Arbitrate state privacy laws

Respondents indicated that action is needed to address the abundant legal issues tied to ISE privacy, in order to further enable HIE and ISE. The Health Information Security and Privacy Collaboration developed tools to assist state-level stakeholders to identify, analyze, and propose solutions to reduce variation in state privacy laws and to enable states to better understand their privacy laws landscape and potential challenges to [ISE and HIE]. ${ }^{12}$

The Harmonizing State Privacy Law Collaborative, a group formed to develop and advance processes to synchronize state laws and eliminate variation, generated four ideologies that are central to harmonizing state laws governing health-information technology. The ideologies are that the laws must be surveyed, organized logically, analyzed in relation to HIE, and evaluated to determine the feasibility of changing or modifying current state law. ${ }^{12}$

Interviewees indicated that in order to begin this corrective process, it is advantageous to promote discussions among state leaders to develop more consistent privacy laws among states. Interview respondents indicated that preparing documents detailing each state's requirements (ie, state law and HIE privacy obligations) could provide a basis to begin and facilitate discussions.

\section{Challenge 2: Differing consent policies}

Much variation exists among states studied concerning patientconsent models. Consent poses a barrier for ISE connectivity, given that states can choose between "opt in", "opt out", "no consent", and hybrid consent models. Despite differing consent policies, interviews revealed that providers have been able to use direct services, but utilizing query-based exchange for some states' systems has been a challenge. Direct exchange is a service that provides the ability to send and receive secure information electronically between care providers to support coordinated care (ie, secure email is the core of direct; often 
referred to as push). ${ }^{14}$ Query-based exchange is a service that provides the ability for providers to find and/or request information on a patient from other providers, often used for unplanned care (ie, providers query to obtain a comprehensive patient record, often referred to as "pull"). ${ }^{14}$

In addition to varying policies, many state consent policies are often lengthy and convoluted, making it difficult for the average person to fully understand the information. ${ }^{11}$ As a result, patients often give consent beyond that which they intend. ${ }^{15}$ In fact, researchers indicate that many patients believe "consenting to privacy" means their patient data will never be shared with anyone at any time. ${ }^{15}$ Interviewees indicated that consumer confusion could be exacerbated if consumers were asked to provide consent in multiple states or through a complex multistate consenting process.

\section{Potential strategies to overcome challenge 2:}

Reconcile consent policies and segment sensitive data

Respondents advised that familiarity with consent policies of other states is important when moving forward with ISE discussions and implementation. The following suggestions derived from our data provide a means to decrease the challenges associated with variations in consent:

- utilize standardized patient-consent forms ${ }^{16}$

- reduce pressure on patients regarding consent to ISE, and encourage meaningful consent through online forums, rather than solely at the point of service ${ }^{15}$

- require all EHR systems to have the capability to manage differing consent models, as indicated in interviews.

Additionally, interviewees conveyed the point that exchange would be easier if consent policies would uniformly limit sensitive categories of patient health data (eg, mental health, HIV/AIDS, and substance abuse). Uniform policies would clearly define rules governing the access and disclosure of sensitive data. Further, interviews indicated that one option would be to require providers to segment sensitive data at their end, and if this were not adhered to, connection would be denied by the state HIE.

In addition, the key informants interviewed recommended additional security measures for sensitive data, which would further build trust between patients and providers. Interviewees noted that certain sensitive data could be blocked from normal query functions, with access only allowed through documentation of additional patient consent.

\section{Challenge 3: Lack of trust among states regarding improper disclosure}

As indicated in interviews, the computerization of patient health records has caused concern regarding privacy and the potential for improper disclosure for unapproved reasons. Providers fear secondary data use may be inconsistent with their HIE's usage policies (eg, use for nontreatment purposes, such as research, marketing, or commercial or business purposes other than paying claims or providing preauthorizations).${ }^{15}$ If this were to occur, patients' trust in ISE and HIE would likely decrease, and the percentage of participating patients could decline.

One participant noted, "The biggest issue is one of trust: does $[\mathrm{X}]$ trust $[\mathrm{Y}]$ to not use the data for unapproved things?" Some leaders expressed their concern regarding improper disclosure, stating:

$[\ldots]$ if data are used for anything other than treatment, the contractual data-sharing agreement has been violated, and the interface will be disconnected $[\ldots]$.

Potential strategies to overcome challenge 3: Create clearly defined agreements and enforcement policies In an effort to strengthen trust among state HIEs and encourage ISE participation across the country, most interviewees stated that it is imperative to utilize clearly defined data use and participation agreements. Interviewees indicated that state-to-state agreements should specifically define what encompasses "treatment", as well as the liability of each state in the event of an improper disclosure. Additionally, to avoid differences in terms and content, interviewees suggested the implementation of a uniform ISE-participation agreement.

Interviewees noted that clearly defined agreements are important; however, without clear enforcement policies that are actually overseen, they are ineffective. The Health Information Technology for Economic and Clinic Health Act provides oversight and certification processes for EHRs; however, there is a need for federal agencies to impose penalties for participant misuse of data. ${ }^{15}$

\section{Challenge 4: Increasing provider buy-in}

Interview discussion indicated that the implementation of HIE and ISE may cause substantial culture shock for some health care providers. First, latent competitive issues mean providers are wary of sharing information, fearing the loss of patient volume and decreased revenue, as indicated by an interviewee. Second, buy-in is difficult when providers are unable to retrieve information quickly. One interviewee revealed, "[...] like any other business, you have to have good technology with the process and procedure put in place $[\ldots]$ ".

Interviewees noted there was a decreased likelihood for continuous use of direct and query-based exchange when 
attempts are unsuccessful for either a local or interstate situation of use.

Potential strategies to overcome challenge 4: Engage key provider organizations in HIE boards and achieve participation in critical mass

One of our participating HIEs established a strong state leadership board to facilitate trust. This HIE felt that strong leadership boards could encourage potential participants to join regional ISE organizations and enhance relationships between existing participants and the HIE.

The ability to produce a large network of participating providers was mentioned as another means to facilitate buy-in. Interviewed leaders shared learned experiences, noting that when health care professionals were able to "pull" or "push" the correct patient data, most if not all of the time participation increased exponentially. In addition, ISE was received more favorably and the number of providers unwilling to deliver patient care without the use of the system increased.

\section{Challenge 5: Costs to update, implement, and participate}

Interviews revealed that the promotion of ISE will be more fully accomplished by "waiving fees [because that] is really critical". However, financial sustainability for ISE is very challenging, because grant funding does not extend past the implementation phase, ${ }^{4}$ and such funding is typically designated for statewide HIEs, not ISE efforts, as pointed out by participants.

Potential strategies to overcome challenge 5: Waive ISE participation fees and increase grant opportunities for ISE

In order to incentivize states to participate in ISE, expansive data are necessary to demonstrate the benefits. Interviews revealed that waiving ISE fees would increase participation and build interstate relationships among HIE boards, but the challenge is funding. Interviewees indicated that they would like to see extended grant-funding periods for their HIEs and funding opportunities that cover ISE efforts. Initial grants, usually of a 2- or 3-year duration, funded only the beginning phases of implementation and not the costs for continued maintenance, which determine whether participation is feasible. ${ }^{4}$ Furthermore, this funding did not often apply to ISE.

\section{Challenge 6: Distrust toward nonprovider participant inclusion}

Prior research reports concern that the inclusion of nonprovider participants, such as third-party payers and insurance companies, could potentially lead to the misuse of patient information for nontreatment purposes outside the scope of patient consent, such as research, marketing, and commercial and business purposes. ${ }^{2}$ Participants echoed this concern, and indicated that this could be further complicated through ISE because states differ in their current provision and allowance of third-party payers. One expressed concern was that a thirdparty payer could gain access to data in a nonparticipant state via their engagement in a state that allowed their participation in the HIE ("back door" access).

Potential strategies to overcome challenge 6: Develop authorization and audit processes and policies

Interviewees suggested that the implementation of an authorization user agreement would assist in the documentation, approval, and monitoring of end users by each ISEparticipating state. The authorization process would mandate individual facilities to verify the completion of Health Insurance Portability and Accountability Act training and other training specific to each end user's role. One particular "trust host model", as termed by interviewees, would charge state HIEs with the task of validating and verifying financial capacity, technical, and security functions, as well as accurately identifying and authorizing end users.

The key informants interviewed collectively expressed the need for audit processes with ISE. As further explained by interviewees, as is the case with some HIEs, participating organizations would be required to perform monthly audits related to all users' exchange activities. The audit process would accomplish the following tasks: first, tracking the total number of "pulls" and "pushes"; and second, tracking the total weekly security overrides of absent patient consent. Security alerts would provide a means to track when health care providers access sensitive data through security overrides without additional required patient consent.

Once again, interviewees indicated state-to-state variations in laws would pose challenges, as states determine the authorization and audit processes to incorporate into ISE agreements. Data sources indicated that the questions that need to be addressed include: 1) Do these agreements cross state lines?; 2) If so, which state would maintain these agreements?; and 3) Which state law will govern these important agreements?

\section{Challenge 7: Lack of cooperation among EHR vendors}

The various technical vendors serving each state may have to work through ways to bridge competing technology systems. One interviewee revealed that one state had 
[...] at least six hospital systems all [with] different software systems $[\ldots]$ normalizing the infrastructure, and distributing the data is just a huge technical challenge [...]

and went on to state that normalizing across ISE HIE systems exacerbated the challenge.

Potential strategies to overcome challenge 7: Facilitate communication among EHR vendors

According to interviewees, vendors should be encouraged to create compatible systems to make ISE connectivity easier. Given that the vendor market is highly competitive, leaders noted that decreasing fees associated with sharing data between two different systems could encourage providers to share data through intrastate and ISE connections.

\section{Challenge 8: Inconsistent patient- identifier conventions}

One major barrier to ISE expressed unanimously by respondents was difficulty adopting a uniform naming convention for patient identity (ie, hyphenated or unhyphenated names and patient locality). For example, one interviewee noted that state HIEs document patients' addresses using state identification or driver's licenses; in contrast, the federal government documents either a patient's residence or the city where the patient is receiving medical treatment. Inconsistency has led to confusion and persistent difficulties when multiple patients have the same identifying information (ie, first and last name, date of birth, zip code) or when documentation as a whole does not match between the state HIE's and federal government. Even if state guidelines exist, cross-state ISE efforts create challenges with identification.

\section{Potential strategies to overcome challenge 8: Establish a uniform patient identifier} Interviewees indicated that there was debate and discussion regarding whether all provider organizations should be required (as a federal mandate) to follow uniform naming conventions to link patients with identifying information more accurately when inputting patient data. In particular, one leader noted that exact locations should be used for the patient's residence and the point of service.

\section{Challenge 9: National HIE status}

Interviewees discussed national HIE efforts with some degree of uncertainty regarding sustainability, structure, and services. Interviewees disclosed their skepticism regarding a realistic time frame for a national HIE to come fully to fruition. This uncertainty creates a challenge to invoke encouragement for the national effort, and also seems to create a platform to build ISE rapport for some, as some interviewees indicated that a national HIE may take many decades to become fully plausible, given the many issues to overcome, and a reason to move forward with ISE effort. In contrast, some states believe ISE connection is unnecessary, given that a national HIE is a future possibility, ${ }^{17}$ and thus will likely wait to connect at a national level.

\section{Potential strategies to overcome challenge 9: Clarity} and actualization

As could be expected, interviewees saw clarity, direction, and actualization of the fate of a national HIE across the country as a first step in addressing this challenge. Efforts toward an interdependent, symbiotic relationship to advance federal- and state-level collective goals were also seen as a way to address this challenge.

\section{Discussion}

\section{ISEs and nationwide HIE}

Many of the challenges outlined herein are not unique to ISE, but can also be applied to broader types of HIEs, such as eHealth Exchange (eHE), the nation's HIE. For example, ISEs can learn from the early efforts of the Office of the National Coordinator and more recent efforts of the Sequoia Project with the development of a common state-level (as opposed to national-level) data-use reciprocal sharing agreement. Such an agreement would eliminate the need to create one-to-one agreements and lessen often cost-prohibitive legal fees. It would further serve to create a common understanding with regard to privacy, consent, and trust, three challenge areas described by interviewees.

Moreover, a reciprocal sharing agreement would create a bridge toward increasing individual state and overall national value in HIE. This value equation has been seen in other "network of networks" configurations, described as group-forming networks, or Reed's law, and changes value such that the whole network (eHE) is other than the sum of the individual networks (ISEs). ${ }^{18}$ This environment exponentially increases the number of health data-exchange transactions that can occur and broadens the geographical reach of individual and collective networks, thereby providing more accurate, current, and comprehensive information at the point of care.

Thoughts about the materialization of a foreseeable national HIE were mixed. Indeed, some interviewees commented that there was a reluctance to create ISEs because 
eventually they will all join the national exchange - eHE. eHE is conceived as a broad-based ISE with a set of standards and policies that will help to improve further the quality and efficiency of health care across the country. ${ }^{17} \mathrm{eHE}$ proposes to work with state, regional, and community HIEs to connect communities on one system, rather than rely on pointto-point testing. ${ }^{19}$ This thinking to "just join eHE" may be short-sighted for two reasons: 1) individual states may have more highly developed data-exchange capabilities providing vertical value, and 2) eHE spans broader geography, providing horizontal value.

Vertical value in terms of statewide HIEs can be exemplified in the challenges and complexities concerning exchange of mental health data. An October 2015 document highlights the progress made and funding received toward facilitating the exchange of mental health patient records in this often complex, costly, and protected patient population. ${ }^{20}$ Such progress at a state level may be limited, challenging at an ISE level, and unable to be adopted at the national level.

Horizontal value in terms of nationwide HIE is by way of volume and reach. eHE currently has over 100 participants, including federal partners, such as the Social Security Administration, the Department of Defense, and the Veterans Health Administration, exchanging records on over 100 million patients. ${ }^{21,22}$ Furthermore, it is estimated that eHE will connect $40 \%$ of the nation's hospitals by the end of $2015,{ }^{21}$ suggesting that eHE has a solid structure and clear identity of service, and that sustainability is on the horizon.

Critical mass for HIE is imminent. While critical mass may be imminent, HIE as a standard of care needs an additional layer of commitment - state exchanges. While it may be the case that HIE is still somewhat abstract to some, use cases such as emergency department use and Social Security disability determination use can help close that gap. As the "use gap" closes, HIE will become a standard of care. Increased HIE use and HIE as a standard of care will lead to more accurate, current, and comprehensive patient data at the point of care. The proximal need for connection across state lines is highlighted by ISE discussions and efforts. Therefore, ISEs can be an important yet less understood component in the overall value-chain structure of nationwide HIE.

\section{Limitations}

Although this study makes strong contributions, limitations do exist. We purposefully collected data from multiple states that share borders with more than one other state (one up to seven borders); however, data collected were limited to seven states: five Midwestern and two non-Midwestern states. We invite future research to extend the study to include representation from states not covered in this manuscript. We also call for future work to leverage this study to contexts outside the USA. Medical mobility is not constrained to the USA, and it is plausible that iterations of the presented findings hold in other situations.

In addition, although the interview process provided a detailed analysis of perspectives from executive leaders, there is still a need to interview actual health care providers one on one (ie, physicians, nurses, etc). As HIE and ISE continue to expand, these data will be more readily available as more providers are able to access and utilize ISE capabilities.

\section{Conclusion}

Accurate and up-to-date patient data are crucial to the health of patients and the sustainability of the health care-delivery system. ${ }^{1}$ A single, robust longitudinal record of patients' health care encounters, testing, and medication (regardless of patient location) is important to providing comprehensive care. Our results indicate that expansion of the ISE of patient health data is necessary to support this goal, because health systems are increasingly crossing state lines, providers are serving patients in different states, and patients are traveling long distances for specialized medical care. The value propositions, indicated by the data supporting this study, demonstrate that ISE is viewed as providing the potential for a robust longitudinal record to address some of the facts and also provides a means for health care providers to learn from one another through enhanced interstate communication and collaboration.

This study articulates a multitude of challenges; however, state HIEs seem to be proactively seeking the means to address these barriers. This study reveals that leaders are hopeful these challenges can be overcome by focusing attention on understanding and negotiating the many state-to-state variations in privacy and consent laws. Furthermore, HIE leaders look to interdependent, symbiotic relationships to advance federal- and state-level collective initiatives toward a single, robust longitudinal record.

We call for future work from the academic, governmental, health care, and technology communities to identify, assess, and enact policies, processes, and advancement to fulfill the promise of an aggregated health record that aligns with the realities of a mobile society and geographic borders.

\section{Acknowledgments}

We would like to acknowledge Missouri Health Connection for their support and encouragement to pursue this study. 
Additionally, we would like to recognize Jennifer Tillman, MHA for her valued contributions to the data-collection process.

\section{Disclosure}

The authors report no conflicts of interest in this work.

\section{References}

1. Office of the National Coordinator for Health Information Technology Privacy and Security Framework Requirements and Guidance for the State Health Information Exchange Cooperative Agreement Program. Washington: US Department of Health and Human Services; 2012.

2. Vest JR, Gamm LD. Health information exchange: persistent challenges and new strategies. J Am Med Inform Assoc. 2010;17(3):288-294.

3. Botts N, Bouhaddou O, Bennett J, et al. Data quality and interoperability challenges for eHealth Exchange participants: observations from the Department of Veteran Affairs' virtual lifetime electronic record health pilot phase. AMIA Annu Symp Proc. 2014;2014:307-314.

4. Kern L, Barron Y, Abramson E, Patel V, Kaushal R. HEAL NY: promoting interoperable health information technology in New York State. Health Aff (Millwood). 2009;28(2):493-504.

5. Kartsakli E, Lalos A, Antonopoulos A, et al. A survey on M2M systems for mHealth: a wireless communications perspective. Sensors (Basel). 2014;14(10):18009-18052.

6. Tennina S, Di Renzo M, Kartsakli E, et al. WSN4QoL: a WSNoriented healthcare system architecture. Int J Distrib Sens Netw. 2014;2014:503417.

7. Dullabh P, Hovey L, Ubri P. Evaluation of the State Health Information Exchange Cooperative Agreement Program: case study synthesis - experiences from five states in enabling HIE. 2013. Available from: https://www.healthit.gov/sites/default/files/casestudysynthesisdocument_2-8-13.pdf. Accessed February 1, 2016.

8. Lee AS, Baskerville RL. Generalizing generalizability in information systems research. Inf Syst Res. 2003;14(3):221-243.

9. Strauss A, Corbin J. Basics of Qualitative Research: Grounded Theory Procedures and Techniques. 2nd ed. London: Sage Publications; 1990.

10. Strategic Health Information Exchange Collaborative. Who we are. Available from: http://strategichie.com/about/who-we-are. Accessed February 1, 2016.
11. McGraw D. Privacy and health information technology. J Law Med Ethics. 2009;37 Suppl 2:121-149.

12. Agency for Healthcare Research and Quality. Health Information Privacy and Security Collaboration. 2014. Available from: https:// www.healthit.gov/sites/default/files/hspl_1_final_rpt.pdf. Accessed February 1, 2016.

13. Christiansen J. Policy Strategies for Advancing Interstate Health Information Exchange: A Report to the State Alliance for e-Health. Washington National Governors Association Center for Best Practices; 2009.

14. Health Information Technology. What is HIE? 2015. Available from: http://www.healthit.gov/providers-professionals/health-informationexchange/what-hie. Accessed July 7, 2015.

15. McGraw D, Dempsey J, Harris L, Goldman J. Privacy as an enabler, not an impediment: building trust into health information exchange. Health Aff (Millwood). 2009;28(2):416-427.

16. Dimitropoulos L, Rizk S. A state-based approach to privacy and security for interoperable health information exchange. Health Aff (Millwood). 2009;28(2):428-434.

17. HIT Consultant. Consortium for national health information exchange launches. 2014. Available from: http://hitconsultant.net/2014/12/15/ consortium-for-national-health-information-exchange-launches. Accessed February 1, 2016.

18. Reed DP. The law of the pack. Harv Bus Rev. 2001;79(2):23-24, 154.

19. Murphy $\mathrm{K}$. What is moving national health information exchange forward? 2014. Available from: https://ehrintelligence.com/2014/02/03/ what-is-moving-national-health-information-exchange-forward. Accessed February 1, 2016.

20. Day A, McDermott H. Breaking through health information exchange barriers in behavioral health. 2015. Available from: http://altarum.org/ health-policy-blog/breaking-through-health-information-exchangebarriers-in-behavioral-health. Accessed October 29, 2015.

21. Sequoia Project [website on the Internet]. Available from: http:// sequoiaproject.org. Accessed October 29, 2015.

22. Perna G. eHealth Exchange grows in numbers, transactions. 2014 Available from: http://www.healthcare-informatics.com/news-item/ ehealth-exchange-grows-numbers-transactions. Accessed March 1, 2014.
Innovation and Entrepreneurship in Health

\section{Publish your work in this journal}

Innovation and Entrepreneurship in Health is an international, peer reviewed, open access journal publishing original research, reports, reviews and commentaries on innovation and entrepreneurship in health. Special focus will be given to the theory, process, and practice of innovation and entrepreneurship by individuals and organizations

\section{Dovepress}

within the health care context globally. The manuscript management system is completely online and includes a very quick and fair peer review system, which is all easy to use. Visit http://www.dovepress.com/ testimonials.php to read real quotes from published authors. 